\section{Un nouveau modèle pour caractériser I'infection par le SARS-CoV-2 et cribler rapidement des molécules thérapeutiques}

\section{L'épithélium respiratoire humain}

Wassim Ouchetto, Coline Moulin, Pierre-Gabriel Roche
École normale supérieure de Lyon, Département de biologie, Master biologie, Lyon, France.

wassim.ouchetto@ens-lyon.fr coline.moulin@ens-lyon.fr pierre-gabriel.roche@ens-lyon.fr

I'utilisation de trois types de cellules présents dans les épithéliums respiratoires humain nasaux ou bronchiques: les cellules ciliées, principales cellules de l'épithélium respiratoire ; les cellules caliciformes, spécialisées dans la synthèse de mucus; et les cellules basales, progénitrices des cellules épithéliales. Ce modèle reproduit ainsi fidèlement la structure de l'épithélium respiratoire humain. II ne contient cependant ni les neurones olfactifs, ni les cellules immunitaires. II permet néanmoins d'étudier la cinétique d'infection par le SARS-Cov-2, et, aussi, l'efficacité de potentielles molécules antivirales, avec un aperçu des processus qui se produisent dans le tractus respiratoire supérieur (le nez), mais aussi dans le tractus inférieur (les bronches). Pizzorno et al. utilisent ce modèle en analysant, successivement, l'infection par le SARSCoV-2 des cellules de l'épithélium, puis les effets de deux traitements potentiels: le diltiazem et le remdésivir. téase à sérine TMPRSS2 (transmembrane serine protease 2), exprimée par les cellules épithéliales. TMPRSS2 permet le clivage de Spike fixée à son récepteur cellulaire, l'ACE2 (angiotensin converting enzyme 2), ce qui conduit alors à la pénétration du virus dans la cellule cible. Cette protéine est exprimée par les cellules de nombreux tissus et organes, tels que les épithéliums nasaux et bronchiques, mais aussi les cellules du système digestif ou les cellules cardiaques [2]. En raison de ce large spectre de tissus cibles potentiels, l'infection par le SARS-CoV-2 est associée à une grande diversité de symptômes. Alors que sa
1 Lignée isolée à partir de cellules épithéliales de rein obtenues à partir d'un singe vert africain. Les cellules de cette lignée ont pour caractéristique d'être incapables d'utiliser la voie normalement activée par l'interféron, une cytokine antivirale normalement produite par les cellules infectées.
Le modèle HAE permet de caractériser le processus d'infection des cellules respiratoires par le SARS-CoV-2

Dans leur article, Pizzorno $A$ et al. ont utilisé une souche de SARS-CoV-2 isolée et séquencée en France et répertoriée dans la base de données GISAID Epi$\mathrm{CoVT}^{\mathrm{M}}$, sous la référence BetaCoV/France/ IDF0571/2020. Les auteurs se sont tout d'abord intéressés à la cinétique de l'infection, dans le modèle $H A \varepsilon$, en titrant le virus produit dans ce système expérimental. La titration consiste ici à mesurer la production de particules virales infec- 


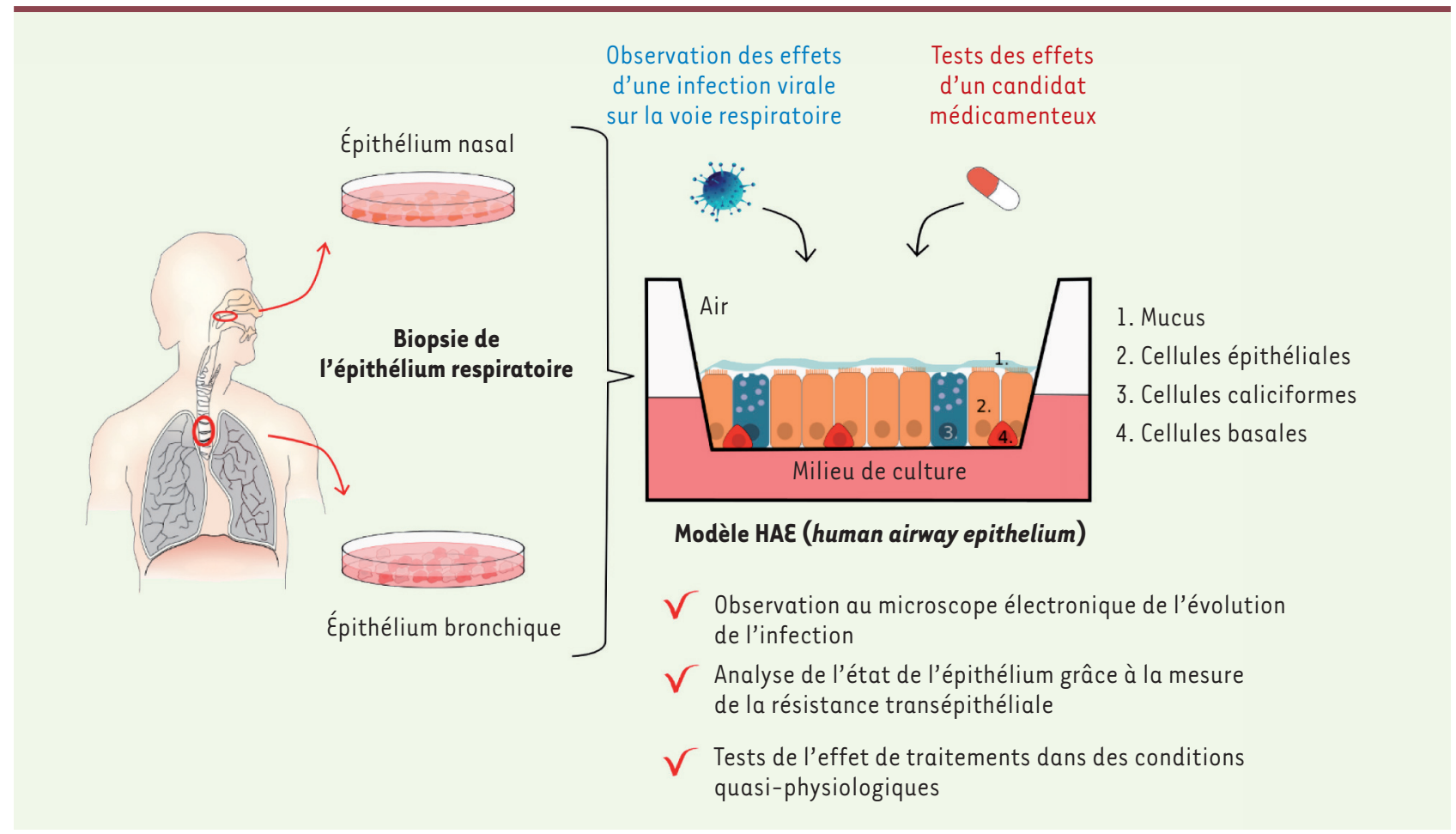

Figure 1. Représentation du modèle HAE (human airway epithelium) et de sa caractérisation par Pizzorno et al. Le modèle HAE repose sur la mise en culture de l'épithélium respiratoire bronchique ou nasal biopsié chez des sujets humains. Ce modèle inclut le mucus et les cellules épithéliales, caliciformes et basales, caractéristiques d'un épithélium respiratoire humain. Cet épithélium reconstitué in vitro, proche de la physiologie humaine, permet de tester différents types d'infections virales ainsi que l'efficacité de potentiels traitements médicamenteux sur l'intégrité de l'épithélium respiratoire.

tieuses par des cellules infectées, en récupérant le surnageant des cellules à différents temps de leur infection, et en l'ajoutant au milieu de culture de cellules sensibles au virus. Le TCID50 (tissue culture infectious dose 50) est une mesure de la quantité de virus infectieux (et cytopathogènes ${ }^{2}$ pour les cellules cibles) présents dans le surnageant. Dans l'épithélium bronchique, le titre viral atteint un plateau entre 24 heures et $72 \mathrm{~h}$, puis diminue. Dans l'épithélium nasal, le titre viral contenu dans le surnageant augmente jusqu'à $96 \mathrm{~h}$ après infection, sans atteindre de plateau. Une différence de cinétique d'infection entre épithéliums nasal et bronchique est ainsi observée, même si cette différence n'est pas statistiquement significative. Les auteurs ont, en parallèle, réalisé une analyse de la charge virale, en utilisant des cellules Vero $\varepsilon 6$, par RT-

\footnotetext{
${ }^{2}$ Le virus détruit les cellules qu'il infecte.
}

qPCR (reverse transcriptase-quantitative polymerase chain reaction) grâce à des sondes élaborées par la School of Public Health de l'université de Hong-Kong. Une corrélation entre charge virale et titre infectieux a ainsi été mise en évidence.

Les auteurs ont alors comparé les titres viraux obtenus dans trois compartiments différents accessibles dans le modèle HAE: le compartiment apical, qui est la zone de contact entre les cellules épithéliales et le mucus; le compartiment intracellulaire; et le compartiment basal, qui se trouve entre les cellules épithéliales et la lame basale. Au niveau nasal apical, les titres viraux augmentent jusqu'à atteindre un pic après $48 \mathrm{~h}$ d'infection. Le virus est également détecté dans le milieu intracellulaire, atteignant un pic après $72 \mathrm{~h}$ d'infection. II est aussi retrouvé dans le compartiment basal, avec une charge virale augmentant jusqu'à 96 heures, ce qui révèle, indirectement, la pro- duction de virus dans le compartiment basal et/ou la rupture de l'intégrité de l'épithélium nasal et bronchique.

Afin de caractériser l'intégrité de I'épithélium, la résistance électrique trans-épithéliale (TEદR, trans-epithelial electrical resistance) a été évaluée à 24,48 et $72 \mathrm{~h}$ après l'infection. Cette résistance est corrélée à l'intégrité physique de l'épithélium [5]. Une diminution importante de la résistance électrique des cellules, révélatrice d'une perte de l'intégrité de la barrière épithéliale, a été observée $48 \mathrm{~h}$ après infection, parallèlement à une augmentation de la charge virale apicale.

Les auteurs ont ensuite utilisé la microscopie électronique pour étudier les effets de l'infection par le SARS-Cov-2 sur les cellules du modèle HAE nasal ou bronchique. Les observations montrent que $48 \mathrm{~h}$ après l'inoculation du virus, les deux types d'épithélium présentent les signes d'une infection bien établie, avec 
une forte production de particules virales par les cellules de l'épithélium. Des vésicules à double membrane, caractéristiques des stades tardifs de l'infection, sont observées dans toutes les cellules de l'épithélium. Ainsi, le modèle HAE permet d'observer les caractéristiques de l'infection de l'épithélium respiratoire par le SARS-CoV-2.

\section{L'infection par le SARS-CoV-2}

déclenche une réponse inflammatoire différente en fonction du type

\section{cellulaire infecté}

Selon de précédentes études, l'infection par le SARS-Cov-2 est généralement associée à un taux élevé de certaines molécules pro-inflammatoires, notamment dans les formes les plus graves de l'infection. Les chercheurs ont donc étudié l'effet de l'infection sur le transcriptome des cellules de l'épithélium HAE nasal et bronchique. Les résultats du séquençage ARN montrent que, $24 \mathrm{~h}$ après l'infection, l'ensemble des cellules de l'épithélium présentent une surexpression de gènes de la réponse inflammatoire tels que ceux codant les interférons de type I ou de type III, ou la chimiokine CXCL2 ( $C-X-C$ motif chemokine ligand 2), et cela, indépendamment du type d'épithélium utilisé. Néanmoins, les résultats montrent que $48 \mathrm{~h}$ après l'infection, une distinction entre la signature transcriptomique des cellules d'épithélium nasal et bronchique est observée, même si celle-ci n'est pas significative. En effet, dans l'épithélium nasal, il existe une tendance à la surexpression des gènes codant les interférons de types I et II (IFNB1, IFNL2, etc.) et le TNF- $\alpha$ (tumor necrosis factor alpha), ainsi que d'autres gènes liés à l'immunité, alors que seuls un petit nombre de gènes impliqués dans l'immunité $(M x l$, ILA, ZBPI, etc.) sont surexprimés dans I'épithélium bronchique. Ces observations suggèrent une potentielle différence entre la réponse innée des voies respiratoires inférieures et celles des voies supérieures.
La combinaison du diltiazem et du remdésivir permet de réduire l'infection et de protéger l'épithélium respiratoire dans le modèle HAE Le remdésivir est une prodrogue nucléotidique (GS-5734) qui est actuellement en cours d'essai pour le traitement de l'infection par le virus Ebola, mais qui semble également pouvoir inhiber la réplication virale du SARSCoV-2 et du MERS dans de nombreux modèles cellulaires et animaux [6]. Le diltiazem est un antagoniste des canaux ioniques $\mathrm{Ca}^{2+}$ voltage-dépendants et il est utilisé comme anti-hypertenseur chez les patients atteints d'arythmie cardiaque [7]. Pizzorno et al. ont montré dans une précédente étude que cette molécule pourrait aussi avoir des effets antiviraux utiles pour traiter le virus de la grippe [7]. En effet, le diltiazem possède la capacité d'induire une réponse interféron antivirale, notamment via les interférons de type I et de type III, par un mécanisme qui n'est pas encore totalement élucidé. La stratégie de la combinaison du diltiazem et du remdésivir repose donc sur le ciblage à la fois de I'ARN du virus par le remdésivir et de l'hôte par le diltiazem.

Pour chaque expérience, le modèle est infecté par le Sars-CoV-2 au jour 0 . En parallèle, le traitement est administré au jour 0 , et répété toutes les 24 h après changement de milieu. Enfin, la charge virale est mesurée à $48 \mathrm{~h}$ et $72 \mathrm{~h}$ postinfection. Chaque expérience est répétée avec différentes concentrations afin de pouvoir déterminer l'IC50, correspondant à la concentration de traitement à partir de laquelle la charge virale est diminuée de plus de $50 \%$. Grâce à ces mesures de I'IC50, Pizzorno et al. ont pu comparer l'efficacité du remdésivir seul ou en association avec le dilitiazem dans les cellules Vero. L'association avec le diltiazem permet de réduire I'IC50 du remdésivir de $0,98 \mu M$ à $0,32 \mu M$ après 48 heures, et de $0,72 \mu \mathrm{M}$ à $0,35 \mu \mathrm{M}$ après 72 heures d'infection. Dans tous les cas, ces concentrations sont grandement inférieures aux concentrations toxiques pour les cel- lules, ce qui fait de cette combinaison thérapeutique une stratégie prometteuse. Dans le modèle $H A \varepsilon$, les deux molécules seules permettent de réduire significativement la charge virale, et l'association des deux molécules est plus efficace encore. Les auteurs ont également mesuré la résistance électrique au niveau nasal et bronchique à $48 \mathrm{~h}$ et $72 \mathrm{~h}$ avec différentes combinaisons de molécules thérapeutiques. À 72 h. Ils ont constaté une amélioration de l'intégrité de l'épithélium après traitement, avec une tendance à la relation dose/effet.

\section{Conclusion}

L'étude de Pizzorno et al. montre qu'in vitro, en utilisant des cellules humaines primaires, l'infection de l'épithélium respiratoire par le SARS-CoV-2 provoque des dommages cellulaires et tissulaires. L'association diltiazem/remdesivir est une potentielle stratégie thérapeutique pour lutter contre la Covid-19 avec des effets montrés in vitro dans le modèle HAE. Ces résultats sont toutefois à mettre en relation avec les récents résultats de l'étude Discovery qui a montré qu'un traitement par remdésivir sans association au diltiazem ne diminue pas la mortalité des patients hospitalisés [8]. Cet article peut en outre être mis en perspective avec les avancées qui ont eu lieu depuis sa publication. Certains chercheurs se focalisent sur le développement de médicaments bloquant les voies d'entrée du virus dans les cellules, tel que le camostat mésylate, aujourd'hui testé en essai clinique. Il s'agit d'un inhibiteur de protéase à sérine qui bloque la protéase TMPRSS2, empêchant ainsi l'entrée virale [9, 10]. D'autres thérapies tentent quant à elles de bloquer le cycle de réplication virale, comme avec le remdésivir que les auteurs étudient dans cet article [6]. Par ailleurs, le développement d'anticorps monoclonaux thérapeutiques dirigés contre la protéine Spike du SARS-CoV-2 est une autre approche explorée. Aujourd'hui, trois traitements 
majeurs ont obtenu des autorisations d'utilisation par la Food and Drug Administration (FDA) : deux bithérapies, casirivimab/imdévimab et bamlanivimab/étésévimab, et une monothérapie avec le bamlanivimab $[11,12]$. Ces approches thérapeutiques sont complémentaires de l'approche vaccinale, aujourd'hui en cours de déploiement autour du globe [13-15]. $\diamond$

Characterization and treatment of SARS-CoV-2 in nasal and bronchial human airway epithelia

\section{LIENS D'INTÉRÊT}

Les auteurs déclarent n'avoir aucun lien d'intérêt concernant les données publiées dans cet article.

\section{RÉFÉRENCES}

1. Amirfakhryan H, Safari F. Outbreak of SARS-CoV2: pathogenesis of infection and cardiovascular involvement. Hellenic J Cardiol 2021 ; 62 : 13-23.
2. Bonny V, Maillard A, Mousseaux C, et al. Covid-19: physiopathologie d'une maladie à plusieurs visages. Rev Med Interne 2020 ; 41 : 375-89.

3. D'Arienzo M, Coniglio A. Assessment of the SARS$\mathrm{CoV}-2$ basic reproduction number, $\mathrm{R} 0$, based on the early phase of Covid-19 outbreak in Italy. Biosafety Health $2020 ; 2$ : 57-9.

4. Pizzorno A, Padey B, Julien T, et al. Characterization and treatment of SARS-CoV-2 in nasal and bronchial human airway epithelia. Cell Rep Med 2020 ; 1 : 100059.

5. Chen S, Einspanier R, Schoen J. Transepithelial electrical resistance (TE६R): a functional parameter to monitor the quality of oviduct epithelial cells cultured on filter supports. Histochem Cell Biol 2015 ; $144: 509-15$.

6. Sheahan TP, Sims AC, Graham RL, et al. Broad-spectrum antiviral GS-5734 inhibits both epidemic and zoonotic coronaviruses. Sci Transl Med 2017 ; 9 : eaal3653.

7. Pizzorno A, Terrier 0, Nicolas de Lamballerie C, et al. Repurposing of drugs as novel influenza inhibitors from clinical gene expression infection signatures. Front Immunol $2019 ; 10: 60$.

8. WHO Solidarity Trial Consortium. Repurposed antivira drugs for Covid-19. Interim WHO solidarity trial results. N Engl J Med 2021 ; 384 : 497-511.

9. Hoffmann M, Hofmann-Winkler H, Smith JC, et al. Camostat mesylate inhibits SARS-CoV-2 activation by TMPRSS2-related proteases and its metabolite GBPA exerts antiviral activity. EBioMed 2021 ; 65 : 103255.
10. University of Aarhus. The impact of camostat mesilate on Covid-19 infection: an investigator-initiated randomized, placebo-controlled, phase lla trial. Clinicaltrials.gov, 2021.

11. Hurt AC, Wheatley AK. Neutralizing antibody therapeutics for Covid-19. Viruses 2021; $13: 628$.

12. Falcone M, Tiseo G, Valoriani B, et al. Efficacy of bamlanivimab/etesevimab and casirivimab/ imdevimab in preventing progression to severe Covid-19 and role of variants of concern. Infect Dis Ther 2021 ; Aug 25 : 1-10. doi: 10.1007/s40121-02100525-4.

13. Meo SA, Bukhari IA, Akram J, et al. Covid-19 vaccines: comparison of biological, pharmacological characteristics and adverse effects of Pfizer/BioNTech and Moderna vaccines. Eur Rev Med Pharmacol Sci $2021 ; 25: 1663-9$.

14. Ramasamy MN, Minassian AM, Ewer KJ, et al. Safety and immunogenicity of ChAdOxl nCoV-19 vaccine administered in a prime-boost regimen in young and old adults (COV002): a single-blind, randomised, controlled, phase 2/3 trial. Lancet $2021 ; 396$ : 1979 93.

15. Voysey M, Costa Clemens SA, Madhi SA, et al. Singledose administration and the influence of the timing of the booster dose on immunogenicity and efficacy of ChAd0xl nCoV-19 (AZD1222) vaccine: a pooled analysis of four randomised trials. Lancet $2021 ; 397$ : 881-91. 